Anaesthesist 2009 · 58:1159-1160

DOI 10.1007/s00101-009-1631-z

Online publiziert: 6. November 2009

๑) Springer Medizin Verlag 2009

\title{
Volatile Anästhetika und linksventrikuläre diastolische Funktion
}

halb möchten wir die Autoren auf zwei klinisch-experimentelle Arbeiten unserer Arbeitsgruppe aufmerksam machen, die in ihrem Leitartikel nicht berücksichtigt worden sind.

In der ersten Studie [2] wurden bei 60 , in 3 Gruppen randomisierten, herzgesunden und nichtprämedizierten Probanden die Auswirkungen von Halothan, Sevofluran (1 MAC) und Propofol ( $4 \mu \mathrm{g} /$ $\mathrm{ml}$ ) auf diastolische Funktion studiert. Diese wurden mithilfe der transthorakalen Doppler- und Gewebedopplerechokardiographie unter Spontanatmung untersucht. Weder Halothan- noch Sevofluranmonoanästhesie hatten einen negativen Einfluss auf die Doppler- und Gewebedopplerindizes der diastolischen Funktion.

Im zweiten Schritt erfolgte die Untersuchung unter mechanischer Ventilation und 1,o sowie 1,5 MAC Halothan bzw. Sevofluran bzw. 4 sowie $6 \mu \mathrm{g} / \mathrm{ml}$ Propofol mithilfe der transösophagealen Echokardiographie. Unter diesen Bedingungen fanden sich leicht abnormale Parameter der diastolischen Funktion in allen 3 Studiengruppen. Sie können weitgehend auf die Interaktion der beatmungsbedingten Reduktion der Vorlast, der propofolinduzierten Vasodilatation und - insbesondere bei den volatilen Anästhetika der negativ-inotropen Wirkung auf den linken Vorhof und Ventrikel zurückgeführt werden.

In der Nachfolgestudie [3] wurden Sevofluran und Propofol bei Patienten mit Aortenklappenstenose und vorbestehender diastolischer Dysfunktion verglichen. Hier vermochte Sevofluran die Dysfunktion sogar zu verbessern. Eine ähnliche, mit Entlastung der linken Kammer einhergehende Verbesserung der Dopplerparameter der diastolischen Dysfunktion durch Isofluran wurde von anderen Autoren auch bei Patienten mit ischämischer Herzerkrankung beschrieben [4].

Unter Propofol ließen sich in unseren Studien keine klinisch relevanten Veränderungen der diastolischen Funktion nachweisen, die sich nicht durch Veränderungen der linksventrikulären Last erklären ließen.

Diese Studien erlauben es, gewisse Lücken im Wissen um die Effekte von Anästhetika auf die diastolische Ventrikelfunktion zu schließen. Es bleiben aber noch manche Fragen unbeantwortet. So bleibt offen, ob sich durch eine perioperative Beeinflussung der Diastole das Auftreten kardialer Komplikationen bei Risikopatienten vermindern lässt.

\section{Korrespondenzadresse \\ Prof. Dr. K. Skarvan \\ Departement Anästhesie \\ Universitätsspital Basel \\ 4031 Basel, Schweiz \\ skarvan@bluewin.ch}

\section{Literatur}

1. Meierhenrich R, Schütz W, Gauss A (2008) Linksventrikuläre diastolische Dysfunktion. Anaesthesist 57:1053-1068

2. Filipovic M, Wang J, Michaux I et al (2005) Effects of halothane, sevoflurane and propofol on left ventricular diastolic function in humans during spontaneous and mechanical ventilation. $\mathrm{Br} J$ Anaesth 94:186-192 und http://www.anaesthesie. ch/publications/echodata.pdf

3. Filipovic M, Michaux I, Wang J et al (2007) Effects of sevoflurane and propofol on left ventricular diastolic function in patients with pre-existing diastolic dysfunction. Br J Anaesth 98:12-18

4. Neuhäuser C, Müller M, Welters I et al (2006) Effect of isoflurane on echocardiographic left-ventricular relaxation indices in patients with diastolic dysfunction due to concentric hypertrophy and ischemic heart disease. J Cardiothor Vasc Anesth 20:509-514
Relevanz der tierexperimentellen Befunde zum Ausdruck zu bringen. Des- 


\section{Erwiderung}

\section{R. Meierhenrich, W. Schütz, A. Gauss} Klinik für Anästhesiologie, Universitätsklinikum Ulm

Wir bedanken uns bei K. Skarvan und seinen Mitarbeitern für den Hinweis auf die von ihnen durchgeführten Untersuchungen, die in unserer Übersicht nicht berücksichtigt wurden und in diesem Kontext Erwähnung finden sollten.

Skarvan et al. beobachteten in einer Studie an herzgesunden Patienten unter dem Einfluss von Halothan eine signifikante Abnahme der dopplersonographisch bestimmten isovolumetrischen Relaxationszeit (IVRT), hingegen keine Veränderung der IVRT unter Sevofluran [1]. In dieser Untersuchung wurde die mithilfe der Gewebedopplerechokardiographie ermittelte frühe myokardiale Bewegung $\left(\mathrm{E}_{\mathrm{a}}\right)$ des lateralen Mitralrings durch beide volatile Anästhetika nicht signifikant beeinflusst. In einer Folgeuntersuchung an Patienten mit Aortenklappenstenose und vorbestehender diastolischer Dysfunktion zeigte sich unter Sevofluran sogar eine Zunahme von $\mathrm{E}_{\mathrm{a}}$ [2]. Die Autoren schlussfolgern aus ihren Ergebnissen, dass Halothan und Sevofluran zu keiner Beeinträchtigung der linksventrikulären Relaxation führen und bei vorbestehender diastolischer Dysfunktion Sevofluran die frühe diastolische Funktion verbessert. Dies steht im Widerspruch zu den Ergebnissen tierexperimenteller Arbeiten, bei denen die Zeitkonstante $\tau$ des isovolumetrischen Druckabfalls invasiv mithilfe eines hochauflösenden Mikromanometerkatheters bestimmt wurde $[3,4,5,6,7]$.

Möglicherweise ist die Diskrepanz der Ergebnisse mit der Lastabhängigkeit der von Skarvan et al. erhobenen dopplersonographischen Parameter zu erklären. Insbesondere die IVRT weist eine ausgeprägte Lastabhängigkeit auf $[8,9,10]$. Die unerwartete Abnahme der IVRT unter Halothan, die auf den ersten Blick eine Verbesserung der linksventrikulären Relaxation vermuten lassen würde, kann beispielsweise auch durch eine Zunahme des linksatrialen Drucks infolge der negativ-inotropen Wirkung von Halothan bedingt sein. Leider ist auch $\mathrm{E}_{\mathrm{a}}$ nicht völlig lastunabhängig, wie man nach Untersuchungen von Sohn et al. anfänglich hätte annehmen können [11, 12, 13]. Neuere Arbeiten haben gezeigt, dass es beispielsweise nach einer Dialysebehandlung zu einem Abfall von $\mathrm{E}_{\mathrm{a}}$ kommt $[14,15]$. Auch die in der Folgestudie von Filipovic et al. [2] beobachtete $\mathrm{Zu}$ nahme von $\mathrm{E}_{\mathrm{a}}$ unter dem Einfluss von Sevofluran ist möglicherweise Folge der negativ-inotropen Wirkung von Sevofluran, zu erkennen an der Abnahme der systolischen Myokardbewegung $S_{a}$ und einem damit verbundenen Anstieg des linksatrialen Drucks. Nicht sicher abzuschätzen ist, inwieweit die durch die volatilen Anästhetika induzierte Nachlastsenkung die IVRT und $\mathrm{E}_{\mathrm{a}}$ beeinflusst hat.

Die Untersuchungen von Skarvan et al. zeigen einmal mehr, wie schwierig es ist, die Auswirkungen einer Substanz auf die diastolische Funktion anhand dopplerechokardiographischer Parameter zu untersuchen, wenn die Substanz gleichzeitig die Lastbedingungen und die Inotropie des Ventrikels verändert.

Skarvan et al. haben richtig bemerkt, dass wir uns zurückgehalten haben, eine Stellungnahme oder gar eine Warnung in Bezug auf den Einsatz von volatilen Anästhetika bei diastolischer Dysfunktion abzugeben. So haben wir explizit darauf hingewiesen, dass letztendlich nicht sicher ist, ob die im Rahmen der tierexperimentellen Arbeiten beschriebene Zunahme der Zeitkonstanten $\tau$ bzw. die Abnahme von - dP/dt überhaupt eine klinische Relevanz im Sinne einer Beeinträchtigung der enddiastolischen Füllung haben. In diesem Zusammenhang ist anzumerken, dass bei allen Betrachtungen über das, was in der frühen Diastole passiert, häufig vergessen wird, „was am Ende dabei herauskommt", nämlich das Ausmaß der enddiastolischen Füllung.

Letztendlich stimmen wir mit Skarvan et al. darin überein, dass aufgrund der derzeitigen Erkenntnisse kein Anlass besteht, bei Patienten mit diastolischer Dysfunktion auf die Anwendung von volatilen Anästhetika zu verzichten.

\section{Korrespondenzadresse PD Dr. Rainer Meierhenrich Klinik für Anästhesiologie Universitätsklinikum Ulm Steinhoevelstraße 9 89075 Ulm rainer.meierhenrich@uniklinik-ulm.de}

Interessenkonflikt. Die korrespondierenden Autoren geben an, dass kein Interessenkonflikt besteht.

\section{Literatur}

1. Filipovic M, Wang J, Michaux I et al (2005) Effects of halothane, sevoflurane and propofol on left ventricular diastolic function in humans during spontaneous and mechanical ventilation. $\mathrm{Br} \mathrm{J}$ Anaesth 94:186-192

2. Filipovic M, Michaux I, Wang J et al (2007) Effects of sevoflurane and propofol on left ventricular diastolic function in patients with pre-existing diastolic dysfunction. Br J Anaesth 98:12-18

3. Pagel PS, Warltier DC (1994) Mechanical consequences of calcium channel modulation during volatile anesthetic-induced left ventricular systolic and diastolic dysfunction. Adv Pharmacol 31:125143

4. Pagel PS, Kampine JP, Schmeling WT, Warltier DC (1991) Alteration of left ventricular diastolic function by desflurane, isoflurane, and halothane in the chronically instrumented dog with autonomic nervous system blockade. Anesthesiology 74:1103-1114

5. Harkin CP, Pagel PS, Kersten JR et al (1994) Direct negative inotropic and lusitropic effects of sevoflurane. Anesthesiology 81:156-167

6. Doyle RL, Foex P, Ryder WA, Jones LA (1989) Effects of halothane on left ventricular relaxation and early diastolic coronary blood flow in the dog. Anesthesiology 70:660-666

7. Humphrey LS, Stinson DC, Humphrey MJ et al (1990) Volatile anesthetic effects on left ventricular relaxation in swine. Anesthesiology 73:731-738

8. Rakowski H, Appleton C, Chan KL et al (1996) Canadian consensus recommendations for the measurement and reporting of diastolic dysfunction by echocardiography: from the Investigators of Consensus on Diastolic Dysfunction by Echocardiography. J Am Soc Echocardiogr 9:736-760

9. Thomas JD, Flachskampf FA, Chen C et al (1992) Isovolumic relaxation time varies predictably with its time constant and aortic and left atrial pressures: implications for the noninvasive evaluation of ventricular relaxation. Am Heart J 124:1305-1313

10. Ishida Y, Meisner JS, Tsujioka K et al (1986) Left ventricular filling dynamics: influence of left ventricular relaxation and left atrial pressure. Circulation 74:187-196

11. Sohn DW, Chai IH, Lee DJ et al (1997) Assessment of mitral annulus velocity by Doppler tissue imaging in the evaluation of left ventricular diastolic function. J Am Coll Cardiol 30:474-480

12. Jacques DC, Pinsky MR, Severyn D, Gorcsan J III (2004) Influence of alterations in loading on mitral annular velocity by tissue Doppler echocardiography and its associated ability to predict filling pressures. Chest 126:1910-1918

13. Firstenberg MS, Levine BD, Garcia MJ et al (2000) Relationship of echocardiographic indices to pulmonary capillary wedge pressures in healthy volunteers. J Am Coll Cardiol 36:1664-1669

14. Vignon $P$, Allot V, Lesage J et al (2007) Diagnosis of left ventricular diastolic dysfunction in the setting of acute changes in loading conditions. Crit Care $11: R 43$

15. Lee TY, Kang PL, Hsiao SH et al (2007) Tissue Doppler velocity is not totally preload-independent: a study in a uremic population after hemodialysis. Cardiology 107:415-421 